\title{
Raman Spectra of Nanodiamonds: New Treatment Procedure Directed for Improved Raman Signal Marker Detection
}

\author{
Raoul R. Nigmatullin, ${ }^{1}$ Dumitru Baleanu, ${ }^{2,3,4}$ Diana Povarova, ${ }^{1}$ Numan Salah, ${ }^{5}$ \\ Sami S. Habib, ${ }^{5}$ and Adnan Memic $^{5}$ \\ ${ }^{1}$ Theoretical Physics Department, Institute of Physics, Kazan (Volga region) Federal University, Kremlevskaya street 18, \\ Kazan 420008, Russia \\ ${ }^{2}$ Chemical and Materials Engineering Department, Faculty of Engineering, King Abdulaziz University, P.O. Box 80204, \\ Jeddah 21589, Saudi Arabia \\ ${ }^{3}$ Department of Mathematics and Computer Sciences, Faculty of Arts and Sciences, Cankaya University, 06530 Ankara, Turkey \\ ${ }^{4}$ Institute of Space Sciences, Magurele, 077125 Bucharest, Romania \\ ${ }^{5}$ Center of Nanotechnology, King Abdulaziz University, P.O. Box 80216, Jeddah 21589, Saudi Arabia
}

Correspondence should be addressed to Adnan Memic; amemic@kau.edu.sa

Received 11 October 2013; Accepted 12 November 2013

Academic Editor: J. A. Tenreiro Machado

Copyright (C) 2013 Raoul R. Nigmatullin et al. This is an open access article distributed under the Creative Commons Attribution License, which permits unrestricted use, distribution, and reproduction in any medium, provided the original work is properly cited.

\begin{abstract}
Detonation nanodiamonds (NDs) have shown to be promising agents in several industries, ranging from electronic to biomedical applications. These NDs are characterized by small particle size ranging from 3 to $6 \mathrm{~nm}$, while having a reactive surface and a stable inert core. Nanodiamonds can exhibit novel intrinsic properties such as fluorescence, high refractive index, and unique Raman signal making them very attractive imaging agents. In this work, we used several nanodiamond preparations for Raman spectroscopic studies. We exposed these nanodiamonds to increasing temperature treatments at constant heating rates $\left(425-575^{\circ} \mathrm{C}\right)$ aiding graphite release. We wanted to correlate changes in the nanodiamond surface and properties with Raman signal which could be used as a detection marker. These observations would hold potential utility in biomedical imaging applications. First, the procedure of optimal linear smoothing was applied successfully to eliminate the high-frequency fluctuations and to extract the smoothed Raman spectra. After that we applied the secondary Fourier transform as the fitting function based on some significant set of frequencies. The remnant noise was described in terms of the beta-distribution function. We expect this data treatment to provide better results in biomolecule tracking using nanodiamond base Raman labeling.
\end{abstract}

\section{Introduction}

Diamond is an important material for both scientific and industrial applications due to its extreme physical, chemical, and biological properties [1]. It is the hardest material known to science and has widespread applications such as cutting and drilling tools, thermal dissipation for electronics, infrared window in harsh environments, filter for surface acoustic wave device, field emission display device, electrochemical sensors in harsh/corrosive environments, biomedical imaging, and so on [2-6]. Diamond exhibits low toxicity and excellent biocompatibility and therefore has great potential as a novel material with potential biomedical applications [6]. There is increasing interest for using nanodiamond as biosensors and fabricating fluorescent nanoscale diamond particles for optical labeling and drug or gene delivery [4-6].

Currently, there are several methods developed for diamond synthesis in general [7-15]. The most common are methods based on high-pressure high-temperature approaches (HPHT) [7] and chemical vapour deposition methods (CVD) $[8,9]$. Other methods include explosive formation (forming detonation nanodiamonds) [10, 11], sonication of graphite solutions (ultrasound cavitation) $[1,12]$, laser ablation [12], high-energy ball milling of HPHT diamond microcrystals [13], autoclave synthesis from supercritical 
fluids [14], chlorination of carbides [15], ion irradiation of graphite [16], electron irradiation of carbon "onions" [17] which were also established.

Diamond's outstanding physical and chemical properties when combined with nanostructure form may lead to hybrid nanodevices with excellent and unique functions and performance $[1,10,14]$. These nanostructures diamonds are often referred as nanodiamonds (NDs) with the expectation of being the next-generation electronic material for specialized nanoelectromechanical systems (NEMS), nanoelectronic devices, and field emission applications [18]. Furthermore, nanodiamonds also have a potential application in biology such as carriers for drugs, genes, or proteins; novel imaging techniques; coatings for implantable materials; biosensors and biomedical nanorobots [19].

Diamond nanoparticles were produced for the first time by detonation method in 1960 [20], but they became popular only by the end of the 1980s [21]. In 1990, a number of important research results led to wider interest in these nanoparticles. For example, colloidal suspended nanodiamonds with particle size in the range of $4-5 \mathrm{~nm}$ became available [22]. Researchers proposed using fluorescent nanodiamonds as a non-toxic alternative to quantum dots for biomedical imaging $[5,6]$. Nanodiamonds were also developed for fabricating magnetic sensors [23]. The nanodiamonds have a good surface chemical reactivity [24-26]; therefore it is possible to tailor the properties of nanodiamonds for use in different applications such as composites [27-31] or attaching drugs and biomolecules when dealing with biological applications [32-34]. In addition, nanodiamonds were found to be less toxic than other carbon nanoparticles such as carbon nanotubes [35-38] and, as a result, are currently being considered for applications in biomedical imaging, drug delivery, and other areas of medicine [19, 38].

Fluorescent nanodiamonds are emerging as a new type of nanomaterial that have great promise for biological applications $[37,39]$. The nanodiamonds that contain a high concentration of nitrogen-vacancy $(\mathrm{N}-\mathrm{V})$ defect centers as fluorophores exhibit several remarkable features such as emission of bright photoluminescence in the extended red region, no photobleaching and photoblinking, and easiness of surface functionalization for specific or nonspecific binding with nucleic acids and proteins [40,41]. The capability of emitting light at $700 \mathrm{~nm}$, where cell autofluorescence signal is low, [19], makes nanodiamonds suitable for cellular imaging application. These excellent photophysical properties, together with the good biocompatibility of the material [5], can enable 3D tracking of a single $35 \mathrm{~nm}$ nanodiamond particle in a live mammalian cell using confocal microscopy [42].

However, ensuring purity of synthesized nanodiamonds is paramount to their application to the field of biomedical imaging in general and Raman tracking in specific. Often other contaminants such as graphite or similar carbon based by-products can be found during the synthesis procedure [7-15]. These impurities can induce fluctuations in the intrinsic Raman signal and therefore they can have negative effects when using the Raman signal as a detection marker.
Furthermore, biological molecules can be adsorbed on the nanodiamond surface providing them a traceable label. Specifically, synthesized nanodiamonds can be characterized by their sharp band using Raman spectroscopy. This band is the characteristic peak of the $\mathrm{sp}^{3}$ structural diamond [10], mostly observed at around $1430 \mathrm{~cm}^{-1}$. However, synthesized nanodiamonds contain considerable amount of graphite, which can be detected by Raman spectrum. The presence of a broad band at around $1590 \mathrm{~cm}^{-1}$ is the inplane vibrations of graphite (G band) $[18,43,44]$. Therefore, Raman spectrum is considered to be a powerful tool to potential tracking of nanodiamonds. The graphite phase can be removed with treatment at relatively lower temperatures in comparison to that of diamonds [44]. This can be seen clearly in the intensity reduction of the corresponding $\mathrm{G}$ band compared with that of diamonds. However, the obtained bands/signals have some noise/fluctuations, which might require further theoretical analysis to observe the actual trends/variations allowing for accurate and improved signal tracking.

In this work, we have prepared three nanodiamond samples, heated at $425^{\circ} \mathrm{C}, 475^{\circ} \mathrm{C}$, and $575^{\circ} \mathrm{C}$, respectively. Next, we obtained Raman spectroscopy spectra for all three heated samples in addition to the untreated "as obtained" nanodiamonds. Our goal was initiating the release of graphite impurities with these temperature treatments, changing the nanodiamond surface/interface properties. We then observed the changes in the Raman spectra based on this treatment. Since even minute amounts of graphite can generate a significant background noise $[43,44]$, novel signal treatment methods are required in order to improve the ability of using the Raman signal as a bioprobe or molecular detection marker. To tackle these challenges we applied the procedure of the optimal linear smoothing (POLS) [45] for the measured Raman spectra of nanodiamonds. Raman spectra for all heat treated and "as obtained" nanodiamonds were used for analysis and comparison in this study. We applied the procedure of POLS in order to eliminate the high-frequency fluctuations and extract the desired trend (smoothed Raman spectrum), aiding in assessing potential application of the Raman tracking signal produced by the nanodiamonds as a detection marker.

\section{Experimental Details}

Detonation nanodiamond particles of size around $6 \mathrm{~nm}$ and purity of more than $98 \%$ were obtained from Nanostructured \& Amorphous Materials Inc., USA (http:// www.nanoamor.com/). These samples were used for further treatments. These nanodiamonds were oxidized at three different temperatures. Specifically we carried out heating at $425^{\circ} \mathrm{C}, 475^{\circ} \mathrm{C}$, and $575^{\circ} \mathrm{C}$ for equal amounts of time, which is $60 \mathrm{~min}$. The heating rate is $10^{\circ} \mathrm{C} / \mathrm{min}$. After heating the sample at the desired temperature it was slowly cooled down to room temperature. Raman spectra were measured and collected using a DXR Raman Microscope, Thermo Scientific, using a $532 \mathrm{~nm}$ laser as the excitation source at $8 \mathrm{~mW}$ power. 


\section{Results and Discussion}

3.1. Application of the Procedure of Optimal Linear Smoothing (POLS). For the measured Raman spectra of nanodiamonds we applied initially the procedure of the optimal linear smoothing (POLS) suggested in [45] in order to eliminate the high-frequency fluctuations and extract the desired trend (smoothed Raman spectrum). We omit the details of this procedure because the POLS have been described earlier in papers [45-48]. In order to decrease the influence of these fluctuations we applied this procedure to the curves that are obtained from the initial ones by numerical integration relatively to its mean value. The usefulness of this procedure was demonstrated earlier in recent paper [47]. In the result of application of the POLS we obtain the smoothed trends that can be analyzed in terms of the secondary Fourier transform (SFT) described below.

The results of the application of the POLS are depicted in Figures 1(a), 1(b), 1(c), and 1(d). Each figure shows the desired trend (smoothed Raman spectrum at the fixed annealing temperature). Usually the optimal value of the smoothing window is located in the interval $[\Delta / 10, \Delta / 1000]$, where $\Delta$ defines the relative length of the initial interval $\Delta=x_{N}-$ $x_{0}$. For simplicity we use as the independent $x$ variable the normalized value of the wavelength $\lambda$; that is, $x=\lambda / 100$. In order to have more reliable result for calculation of the value of the optimal smoothing window, we used as an independent criterion the behavior of the generalized Pearson correlation function (GPCF). The GPCF (based on the statistics of the fractional moments [49]) was introduced previously in paper [50] and it is determined as

$$
\mathrm{GPCF}_{p}=\frac{\mathrm{GMV}_{p}(1,2)}{\sqrt{\mathrm{GMV}_{p}(1,1) \cdot \mathrm{GMV}_{p}(2,2)}},
$$

where the generalized mean value function (GMV-function), in turn, is defined as

$$
\begin{array}{r}
\operatorname{GMV}_{p}(k, l)=\left(\frac{1}{N} \sum_{j=1}^{N}\left|\mathrm{nrm}_{j}(k) \cdot \mathrm{nrm}_{j}(l)\right|^{\left.\operatorname{mom}_{p}\right)^{1 / \mathrm{mom}_{p}},}\right. \\
\operatorname{mom}_{p}=\exp \left(\operatorname{Ln}_{p}\right), \quad L n_{p}=m n+\left(\frac{p}{P}\right) \cdot(m x-m n), \\
p=0,1, \ldots, P .
\end{array}
$$

Here the values $k$ and $l$ numerate a couple of compared sequences. At $\operatorname{mom}_{p}=1$ expression (2) coincides with the conventional definition of the Pearson correlation coefficient. The normalized sequences located in the interval $0<$ $\operatorname{nrm}(y)<1$ are determined below by expression (3). The value mom $_{p}$ determines the current moment from the interval $[0, P]$. The value $P$ determines the final value of the function $\operatorname{Ln}_{p}$ located in the interval $[m n, m x]$. The values $m n$ and $m x$ define correspondingly to the limits of the moments in the uniform logarithmic scale. In many practical cases, these values are chosen as $m n=-15$ and $m x=15$ and $P$ is chosen as integer value from the interval [50-100].
This empirical choice is related to the fact that the transition region of the random sequences considered expressed in the form of the GMV-functions are concentrated in the interval $\operatorname{Ln}_{p} \in[-5,5]$ and the extended interval $[-15,15]$ is taken for showing the limiting values of this function in the space of moments. The initial sequences are chosen in that way: the minimum of the GMV-function coincides with zero value while the maximal value of this function coincides with $\max \left(\operatorname{nrm}_{j}(y)\right)$. In (2) the random sequences are supposed to be normalized to the unit value in accordance with expression

$$
\begin{gathered}
\text { (A) } \operatorname{nrm}_{j}(y)=\frac{y_{j}^{(+)}}{\max \left(y_{j}^{(+)}\right)}-\frac{y_{j}^{(-)}}{\min \left(y_{j}^{(-)}\right)}, \\
y_{j}^{( \pm)}=\frac{1}{2}\left(y_{j} \pm\left|y_{j}\right|\right), \\
\text { (B) } \operatorname{nrm}_{j}(y)=\frac{\Delta y_{j}}{\max \left(\Delta y_{j}\right)}, \quad \Delta y_{j}=y_{j}-\min \left(y_{j}\right), \\
j=1,2, \ldots, N, \quad 0<\operatorname{nrm}(y)<1 .
\end{gathered}
$$

Here the set $y_{j}$ defines the initial random sequence that can contain the trend or can be compared with another sequence without trend. The symbol $|\cdots|$ and index $j$ determine the absolute value and number of the measured points, correspondingly. The second case (B) in (3) corresponds to the case when the initial sequence is completely positive. If the limits $m n$ and $m x$ in (2) have opposite signs and accept sufficiently large values then the GPCF function has two plateaus equaled one at small numbers of $m n$ (i.e., $\mathrm{GPCF}_{m n}=$ 1) and another limiting value $\mathrm{GPCF}_{m x}$ depends on the degree of correlation between the random sequences compared. This right-hand limit (defined as $L$ ) is located between two values:

$$
M \equiv \min \left(\mathrm{GPCF}_{p}\right) \leq L \equiv \mathrm{GPCF}_{m x} \leq 1
$$

The appearance of two plateaus implies that all information about possible correlations is complete and further increasing of the limiting numbers $(m x, m n)$ figuring in (7) is not necessary. The numerous test calculations show that the high degree of correlations between two random sequences compared is observed when $\mathrm{GPCF}_{m x}$ coincides with the unit value, while the lowest correlations are observed when GPCF $_{m x}$ is equaled to its minimal value $(M)$. This simple observation having general character for all random sequences allows us to introduce new correlation parameter, (CC) complete correlation-factor, which is determined as

$$
\mathrm{CC}=\left(\begin{array}{c}
L \\
M
\end{array}\right) \cdot\left(\frac{L-M}{1-M}\right) .
$$

We would like to stress here that this factor is determined on the total set of the fractional moments located between $\exp (m n)$ and $\exp (m x)$ values (see definition (2)). As it has been remarked above, in practical calculations for many cases it is sufficient to put $m n=-15$ and $m x=+15$, correspondingly. The upper row in (10) is referred to the CCL 


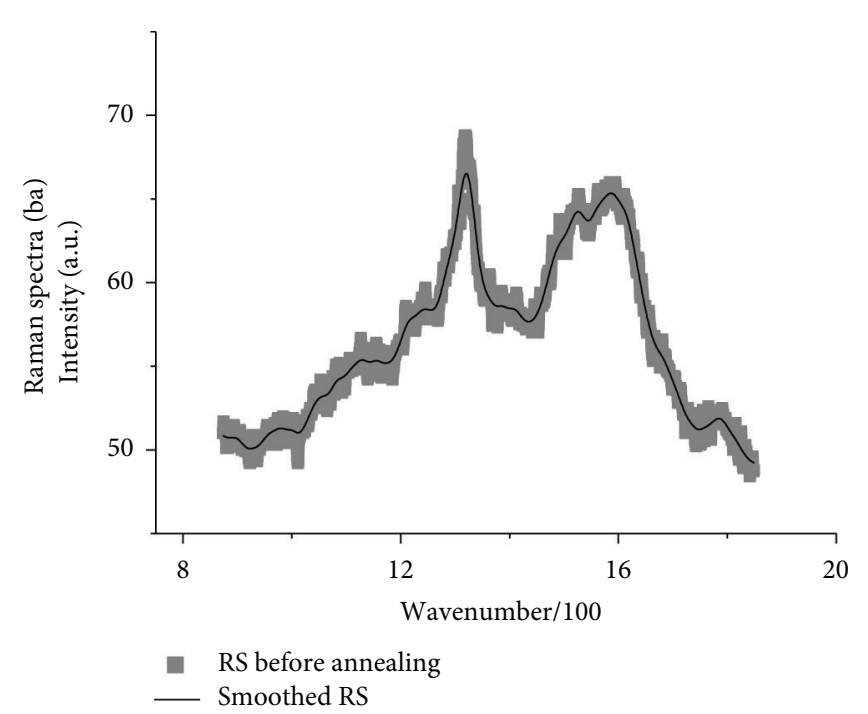

(a)

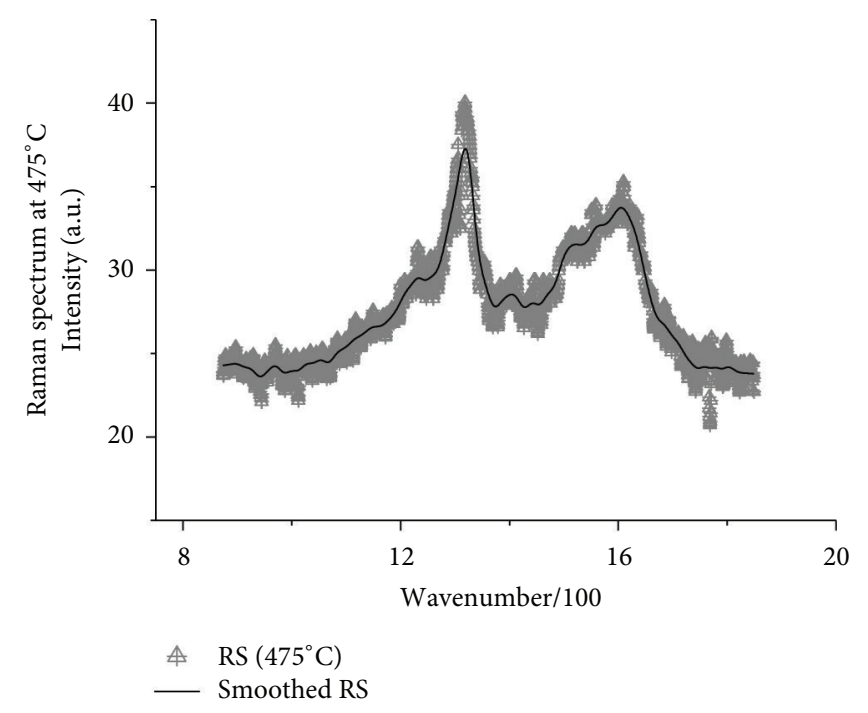

(c)

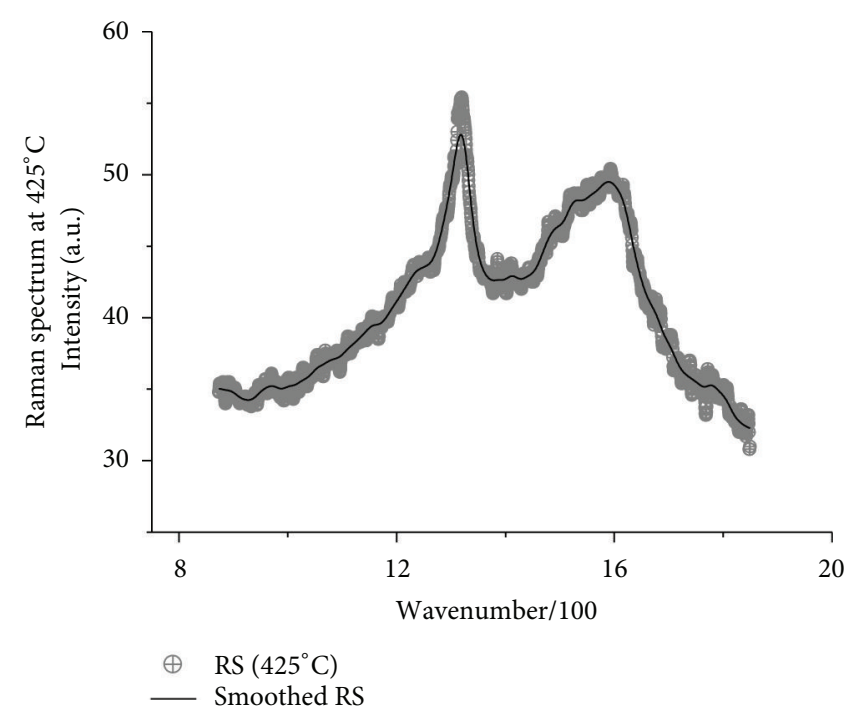

(b)

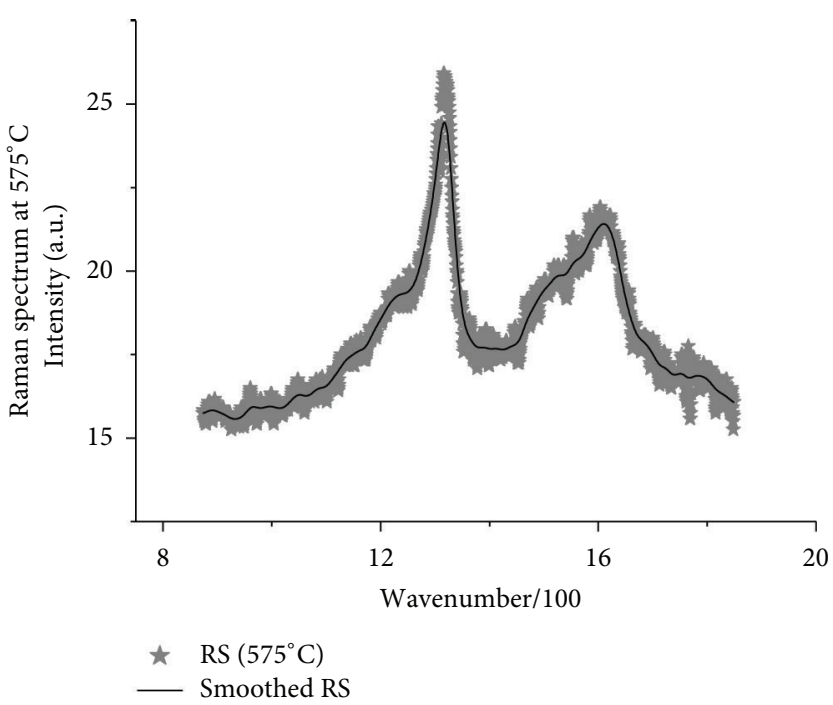

(d)

Figure 1: (a) The initial Raman spectrum (before annealing (ba) marked by grey squares) and its optimal trend (black solid line) obtained by application of the POLS. The optimal value of the smoothing window is shown in Figures 2(a) and 2(b). (b) The initial Raman spectrum (grey crossed points) measured at $425^{\circ} \mathrm{C}$ and its optimal trend (black solid line) obtained by application of the POLS. The optimal value of the smoothing window is shown in Figures 2 (a) and 2(b). (c). The initial Raman spectrum (grey crossed triangles) measured at $475^{\circ} \mathrm{C}$ and its optimal trend (black solid line) obtained by application of the POLS. The optimal value of the smoothing window is shown in Figures 2(a) and 2(b). (d) The initial Raman spectrum (grey stars) measured at $575^{\circ} \mathrm{C}$ and its optimal trend (black solid line) obtained by application of the POLS. The optimal value of the smoothing window is shown in Figures 2(a) and 2(b).

(with respect to the limiting value $L$ ) while the low row determines the factor associated with the minimal value $M$. In practical calculations, both factors are useful for analysis but the CCL-factor is less sensitive to the strong correlations (or small perturbations of the initial sequence) in comparison with the CCM-factor. In addition, we want to stress also the following fact. This statistical parameter does not depend on the amplitudes of the random sequences compared. The pair random sequences compared should be normalized to the interval: $0 \leq\left|y_{j}\right| \leq 1$. It reflects the internal structure of correlations of the compared random sequences based presumably on the similarity of the probability distribution functions that are not known in many cases. In order to see how the highfrequency fluctuations are separated from the low-frequency fluctuations (which is conventionally defined as a trend) we put as initial function initial Raman spectrum $(\operatorname{RS}(d))$ where $d$ determines the initial RS before annealing (ba, $d=0$ ) and after annealing measured at three temperatures $\left(425^{\circ} \mathrm{C}\right.$, 


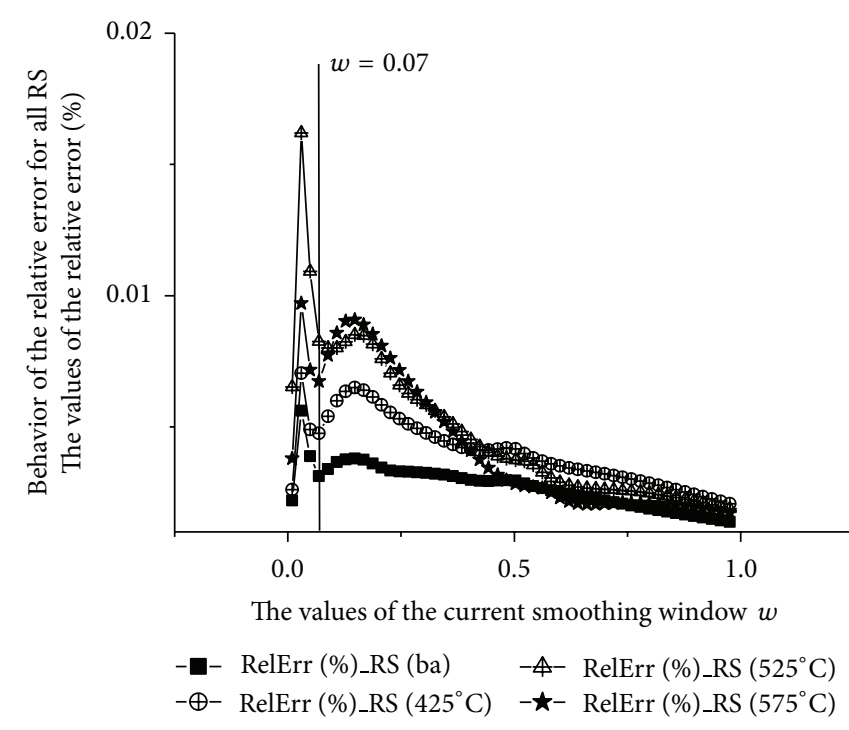

(a)

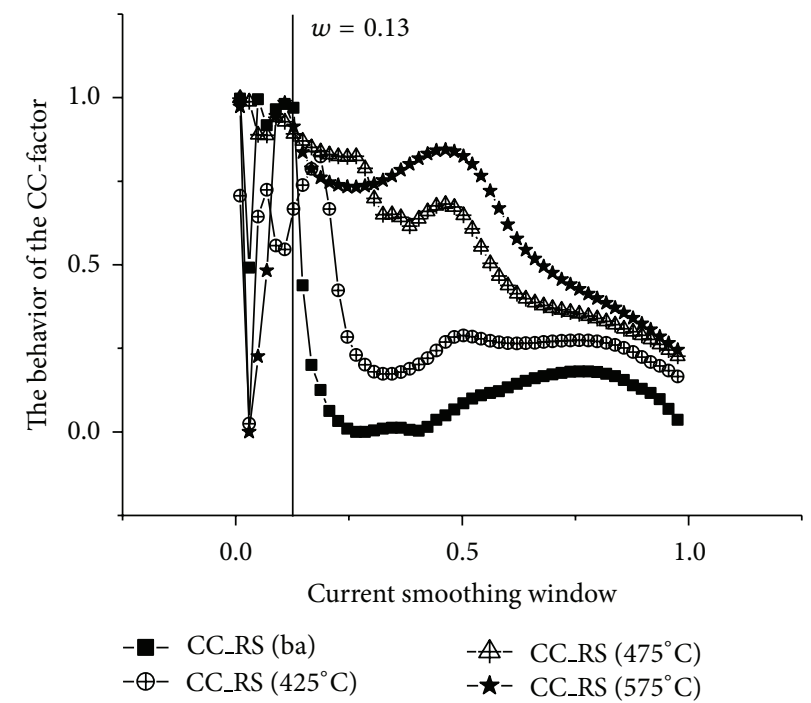

(b)

Figure 2: (a) The behavior of the relative error for all Raman spectra with respect to the current smoothing window. The value of the first minimum is equaled approximately 0.07. (b) The behavior of the complete correlation factor (expression (7)) is shown for all Raman spectra data. The value of the smoothing window $w=0.13$ shows approximately the boundary dividing the high-frequency fluctuations from the low-frequency fluctuations (trend). From these two plots we chose the mean value of the smoothing window $w=0.1$ which is identified as the optimal one.

$d=1),\left(525^{\circ} \mathrm{C}, d=2\right)$, and $\left(575^{\circ} \mathrm{C}, d=3\right)$, correspondingly. As a second sequence we use the smoothed spectra obtained at the fixed value of the current smoothing window $w_{k}$ from the interval $\left[w_{\min }=\Delta / 1000, w_{\max }=\Delta / 10\right]$. It is calculated as

$$
\begin{gathered}
y_{j}\left(d, w_{k}\right)=\operatorname{Gsm}\left(x, y, w_{k}\right) \\
\equiv \frac{\sum_{j=1}^{N} K\left(\left(x_{i}-x_{j}\right) / w_{k}\right) y_{j}(d)}{\sum_{j=1}^{N} K\left(\left(x_{i}-x_{j}\right) / w_{k}\right)}, \\
K(x)=\exp \left(-\frac{x^{2}}{2}\right), \\
\operatorname{GPCF}_{p}\left(\operatorname{nrm}(d), y \operatorname{nrm}\left(d, w_{k}\right)\right) \rightarrow \operatorname{CCM}_{k}=\frac{\left(L_{k}-M_{k}\right)}{1-M_{k}} .
\end{gathered}
$$

These expressions combined together allow calculating the complete correlation factor $\mathrm{CCM}_{k}$ as a function of the current smoothing window $w_{k}$. This value $w_{\text {bound }} \approx w_{\text {opt }}$ separates the correlations evoked by high-frequency fluctuations from low-frequency ones. This observation helps to find some additional arguments that justify the selection of the optimal trend in accordance with expressions (7). This additional criterion is important especially in cases when the first local minimum in the relative error function in expression (8) is not clearly expressed:

$$
\begin{gathered}
\widetilde{y}_{w^{\prime}}=\operatorname{Gsm}\left(x, \tilde{y}_{w}, w^{\prime}\right), \quad w^{\prime}<w, \\
\min (\operatorname{Re} \text { lErr })=\left[\frac{\operatorname{stdev}\left(\left|y_{w^{\prime}}-y_{w}\right|\right)}{\operatorname{mean}\left(\left|y_{w}\right|\right)}\right] \cdot 100 \%,
\end{gathered}
$$

$$
\begin{gathered}
\operatorname{stdev}(y)=\left(\frac{1}{N} \sum_{j=1}^{N}\left(\Delta y_{j}\right)^{2}\right)^{1 / 2}, \Delta y_{j}=y_{j}-\operatorname{mean}(y) \\
\operatorname{mean}(y)=\frac{1}{N} \sum_{j=1}^{N} y_{j}
\end{gathered}
$$

That is why this optimal trend can be defined as the pseudofitting function which divides the high-frequency fluctuations from a trend. The behavior of the functions (8) and (7) is shown in Figures 2(a) and 2(b), correspondingly.

3.2. Application of the Secondary Fourier Transform as the Fitting Function. One can use the secondary Fourier transform as the fitting function based on some significant set of "frequencies." In accordance with conventional definition we determine this transformation of the second order as

$$
\begin{aligned}
& \operatorname{SmRS}\left(x_{j} ; d\right) \cong F\left(x_{j}\right) \\
&=A_{0}^{(d)}+\sum_{k=1}^{K} {\left[\operatorname{Ac}_{k}^{(d)} \cos \left(2 \pi k \frac{x_{j}}{L_{d}}\right),\right.} \\
&\left.\quad+\mathrm{As}_{k}^{(d)} \sin \left(2 \pi k \frac{x_{j}}{L_{d}}\right)\right], \\
& \omega_{k}=2 \pi k\left(\frac{1}{L_{d}}\right) .
\end{aligned}
$$


We suppose that the characteristic inverse length $L_{d}(d=$ $0,1,2,3$ is the type of the RS defined above) coincides with the maximal length of the interval $\Delta=x_{N}-x_{0}=L$ ( $x$ defines the normalized wave-number/100) and is measured in the same units as wavelength $\lambda$. If the value $L$ is supposed to be known then the unknown decomposition coefficients $A c_{k}$ and $A s_{k}$ can be found by the linear-least square method (LLSM) and the limiting value $K$ can be found from the condition of minimization of the value of the relative error:

$$
\begin{aligned}
1 \%<\operatorname{Re} 1 \operatorname{Err}= & {\left[\frac{\operatorname{stdev}\left(\left|\operatorname{SmRS}\left(x_{j}, d\right)-F\left(x_{j}, K\right)\right|\right)}{\operatorname{mean}\left(\left|\operatorname{SmRS}\left(x_{j}, d\right)\right|\right)}\right] } \\
& \cdot 100 \%<10 \%,
\end{aligned}
$$

which should be located in the reasonable interval (1-10\%) of the calculated errors. It is interesting to note that this new interpretation of the discrete Fourier transform as the fitting function of the initial signal does not coincide with conventional presentation of the Fourier transform as presentation of the function in the frequency space. The coefficients $\mathrm{Ac}_{k}$ and $\mathrm{As}_{k}$ found in the result of the application LLSM do not coincide with decomposition coefficients found in the result of application of the conventional program based on the fast Fourier transformation (FFT) and its modifications. Initially, we suppose simply that the period is found from the condition $\Delta=x_{N}-x_{0}=L$. But further investigations show that this supposition can be corrected in order to decrease the value of the fitting error. This observation is illustrated by the plot depicted in Figure 3. After selection of the optimal value of $L$ one can fit function (9) to the smoothed Raman spectra for nanodiamonds obtained in Section 3.1. In order to compare them with each other we chose the limiting value of modes $K$ (number of components figuring in (9)) equaled 40. The results of the fitting of the smoothed Raman spectra corresponding to different annealing temperatures are shown in Figure 4. The additional fitting parameters are shown in Table 1. We want to stress here that in the absence of the microscopic model the application of the secondary Fourier transform allow us to reduce the 2025 measured points for each spectrum to 40 fitting parameters $19\left(A c_{k}\right)+19\left(A_{k}\right)$ amplitudes figuring in decomposition (9) plus free fitting constant $A_{0}^{(d)}$ and $L_{d}$ for 4 types of Raman spectra. This reduced presentation with the help of the secondary Fourier transform is very convenient when the actual microscopic model describing the vibrations in nanodiamond dusts is absent but the barest necessity of description these RS exits. So, in brief, with the help of secondary Fourier transform we can reduce the Raman spectra to its amplitude-"frequency" response (AFR). Schematically, it can be written as

$$
\operatorname{Spectrum}(\lambda, N) \longrightarrow \operatorname{AFR}\left(\operatorname{Ac}_{k}, \operatorname{As}_{k}, K\right), \quad K \ll N
$$

So, analysis of the Raman spectra can be based on the additional analysis of the amplitude-"frequency" responses (AFR) (we should notice again that in our case a "frequency" coincides with the value $\omega_{k}=2 \pi k / L$ ). This set of "frequencies" giving the acceptable accuracy should be located in the

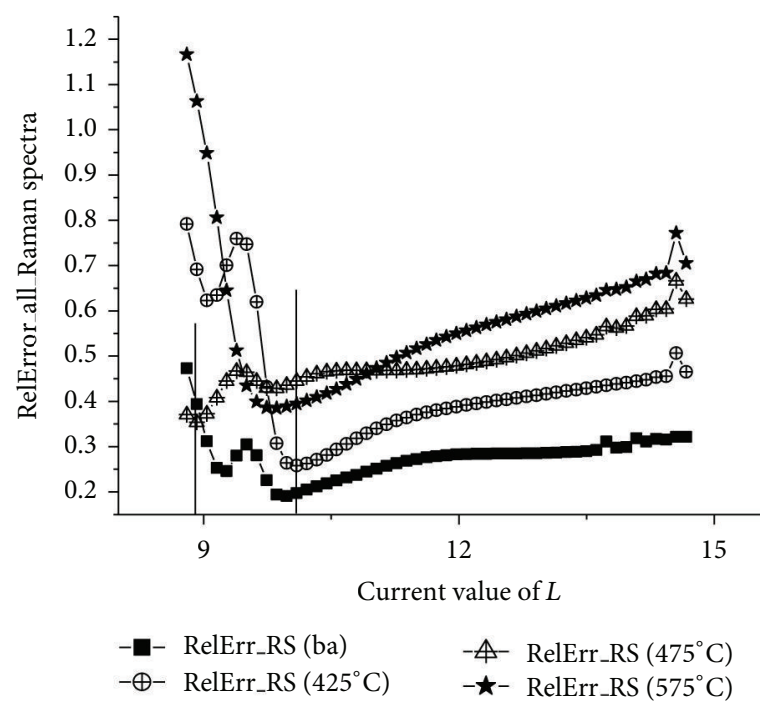

FIGURE 3: This plot clearly demonstrates that the relative fitting error can be essentially decreased (on the half-order of magnitude at least) if the optimal $L$ in decomposition (9) can be found. The initial points located on the left-hand side correspond to the initial selection of $L$ from the condition $x_{N}-x_{0}=L$. The minimal (optimal) values of $L$ are collected in Table 1 . The bold vertical lines show the limits of the optimal values of $L$. All these plots are obtained for the limiting value of $K=40$ in (9), which is chosen as the optimal for the fitting purposes.

interval $\left[\omega_{\min }=2 \pi / L, \omega_{\max }=2 \pi K / L\right]$. So, we show that the secondary application of the Fourier transform (used as a fitting function of the initial signal) gives new possibilities for the interpretation of the smoothed RS data in terms of the reduced set of the calculated amplitudes $\mathrm{Ac}_{k}$ and $\mathrm{As}_{k}$. Figure 4 demonstrates the fitting of the smoothed Raman spectra in the frame of this secondary Fourier analysis. The variations of the decomposition parameters $\left(\mathrm{Ac}_{k}, \mathrm{As}_{k}\right)$ together with its modulus for all Raman spectra are shown in Figures 5(a), 5(b), and 5(c). Other additional parameters are collected in Table 1.

3.3. "Reading" of the Remnant Noise in Terms of the BetaDistribution Function. Usually, analysis of experimental data is finished after selection of the proper fitting function corresponding to some model and the "remnants" defined as the difference between the spectra analyzed and its fitting function is usually not analyzed. However, recent achievements associated with detection of the universal distribution function for the strongly correlated sequences allow realizing the fit of the remnants (noise) to the fitting function corresponding to beta-distribution [51]:

$$
\mathrm{Jb}(x)=A\left(x-x_{0}\right)^{\alpha}\left(x_{N}-x\right)^{\beta}+B
$$

and express quantitatively the remnant noise in terms of 4 fitting parameters $(A, B, \alpha$, and $\beta)$ only. This possibility gives a unique chance to compare the remnant functions with each other quantitatively. In order to obtain the bell-like curve 
TABLE 1: The additional parameters of the secondary Fourier fitting.

\begin{tabular}{lcccc}
\hline $\begin{array}{l}\text { Number of Raman } \\
\text { spectrum }\end{array}$ & $\begin{array}{c}\text { The value of } L_{\text {opt }} \text { from } \\
\text { decomposition }(9)\end{array}$ & $\begin{array}{c}\text { The value of } A_{0} \text { (d) from } \\
\text { decomposition (9) }\end{array}$ & $\begin{array}{c}\text { The value of the fitting } \\
\text { error (\%) }\end{array}$ & $\begin{array}{c}\text { Pearson correlation } \\
\text { coefficient }(\text { PCC) }\end{array}$ \\
\hline RS (ba), $d=0$ & 9,97637 & 56,4327 & 0,19051 & 0,99976 \\
RS (425C) $d=1$ & 10,0937 & 40,5278 & 0,25801 & 0,9998 \\
RS (475C) $d=2$ & 8,92004 & 28,021 & 0,35399 & 0,9996 \\
RS (575C) $d=3$ & 9,859 & 18,0758 & 0,38478 & 0,99938 \\
\hline
\end{tabular}

It is interesting to note that the values in columns 3 and 4 have the monotone behavior and so this peculiarity can be used for calibration purposes.

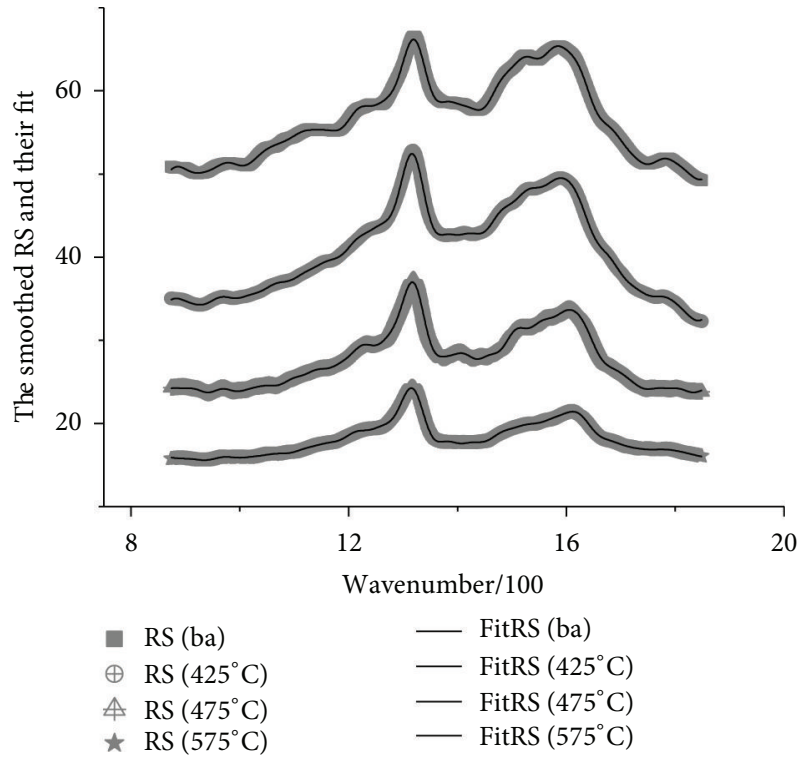

Figure 4: Here we show the results of the fitting of the secondary Fourier transform to the smoothed Raman spectra. The value of a free constant (from (9)) and other parameters $\left(L_{\text {opt }}\right)$ are collected in Table 1.

from the remnant function it is necessary to do the following steps.

(S1) Calculate the difference

$$
\operatorname{Rmf}(x, d)=\operatorname{Spectrum}(x, d)-\operatorname{Fit}(x, d),
$$

where $\operatorname{Rmf}(x)$ defines the remnant function, $\operatorname{Spectrum}(x)$ defines the smoothed spectrum, and Fit $(x)$ is associated with the corresponding fitting function. Index $d$ as before defines the type of the Raman spectra.

(S2) Then, it is necessary to sort the amplitudes of the $\operatorname{Rmf}(x)$ in descending order $\left(y_{1}>y_{2}>\cdots>y_{N}\right)$, subtract its mean value, and numerically integrate the rest:

$$
\begin{gathered}
D y_{j}=y_{j}-\operatorname{mean}(y), \\
J y_{j}=J y_{j-1}+0.5 \cdot\left(x_{j}-x_{j-1}\right) \cdot\left(D y_{j}+D y_{j-1}\right), \\
J y_{0}=0, \quad j=1,2, \ldots, N .
\end{gathered}
$$

In the results of these manipulations we obtain the bell-like curve that can be fitted to expression (13) with the help of Eigen-coordinates (ECs) method [52]. Figure 6 demonstrates these two steps transforming the desired remnant function corresponding to the Raman spectrum $(d=0)$. In Figure 7 we show the final fit of all bell-like curves to the fitting function (12). We want to stress here that the ECs method [52] allows fitting the sufficient number of the measured points (2025) and reducing all fit to the conventional LLSM. The fitting parameters are collected in Table 2. Analysis of these curves shows clearly that the distribution of their heights with respect to increasing of the annealing temperature is not monotonic. The highest curve belongs to the annealing spectrum with $475^{\circ} \mathrm{C}$. Then the curves belonging to RS before annealing and $425^{\circ} \mathrm{C}$ follow to monotone behavior and the lowest curve belongs to the annealing temperature $475^{\circ} \mathrm{C}$.

\section{Conclusions}

The use of nanodiamonds as potential labels, probes, or tracers based on Raman specific detection is of great biological importance. In our study, we used three heat treated samples and compared them to the "as obtained" nanodiamond Raman signal. We showed that inducing the graphite heat release changes the nanodiamond surface interface that affected the Raman spectrum. It is clear from our data that these Raman signals were in need of data treatment due to their high-frequency fluctuations that could prove problematic in noisy cellular environments. Based on the four Raman spectra we are able to extract signal trends in the Raman signal resulting from the heat induced changes and finding the optimal for Raman signal fitting. Therefore, this can aid noise removal that is beneficial for future Raman based signal tracking based on nanodiamond particles in biological environments. In general, we were able to improve access to Raman spectroscopic mapping and signal tracking. We realized this procedure by application of the additional Fourier analysis using the finite Fourier decomposition as an additional fitting function (see expression (9)). This simple procedure helps decrease the number of the fitting parameters and gives a possibility to compare the spectra with each other. We demonstrate also how to read a remnant noise after elimination of the smoothed spectra. It helps also compare the noise in terms of the fitting parameters describing beta-function. Definitely, these new innovation elements can be applied in different nanotechnologies at analysis of small amount of materials, when the influence of 


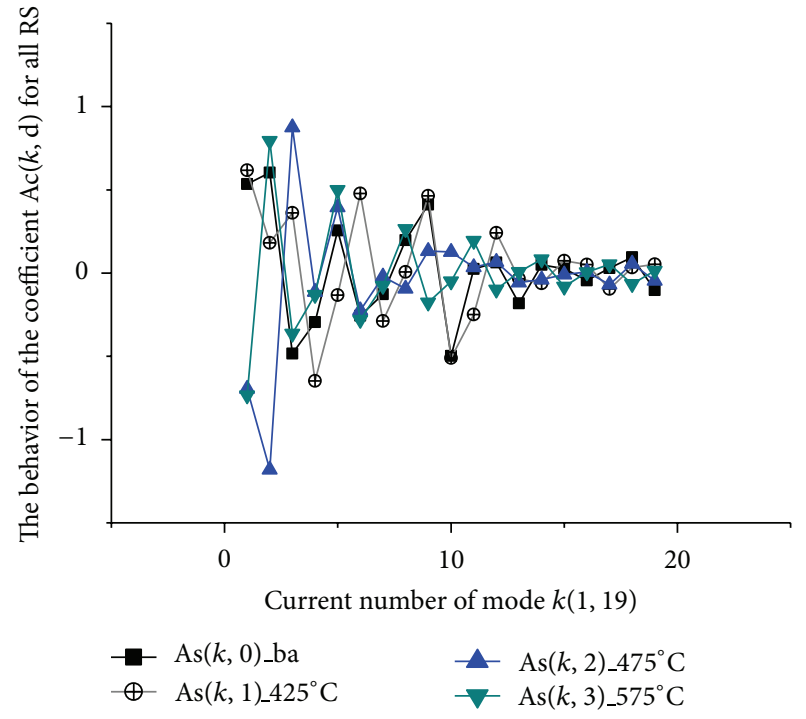

(a)

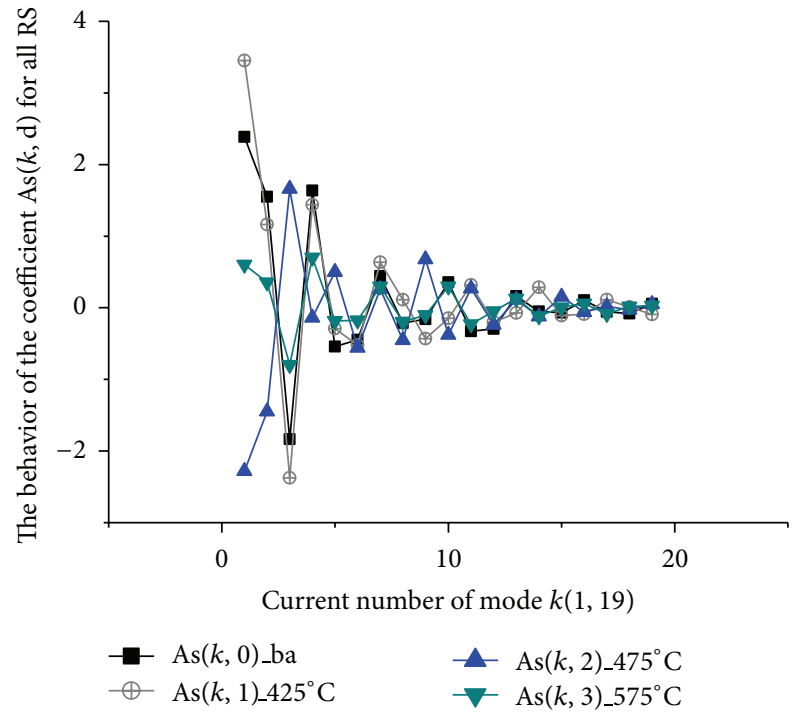

(b)

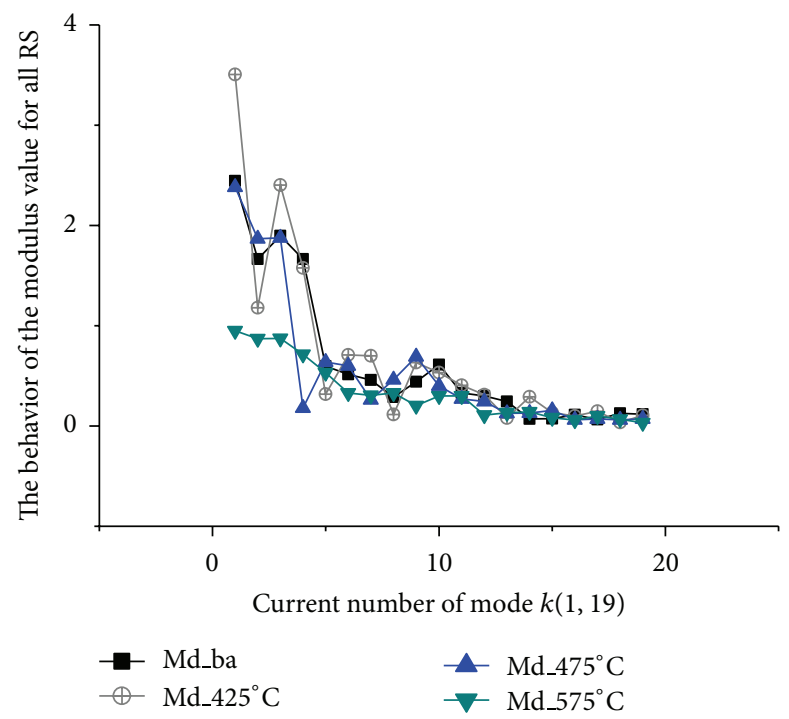

(c)

Figure 5: (a) Here we show the variations of the constant $\operatorname{Ac}(k, d)$ figuring in decomposition (9) for all 4 Raman spectra analyzed. (b) The variations of the constant $\operatorname{As}(k, d)$ from decomposition (9) for all 4 Raman spectra analyzed. (c) Here we demonstrate the variations of the modulus $\left(\operatorname{Ac}(k, d)^{2}+\operatorname{As}(k, d)^{2}\right)^{1 / 2}$ for all 4 Raman spectra analyzed.

TABLE 2: The fitting parameters of all beta-distribution functions.

\begin{tabular}{|c|c|c|c|c|c|}
\hline $\begin{array}{l}\text { Number of Raman } \\
\text { spectrum }\end{array}$ & $A, B$ & $\alpha$ & $\beta$ & $\begin{array}{l}x_{\max } \\
y_{\max }\end{array}$ & RelErr (\%) \\
\hline $\begin{array}{l}\mathrm{RS}(\mathrm{ba}), \\
d=0\end{array}$ & $\begin{array}{c}0,18165 \\
-0.01731\end{array}$ & 0,76645 & 0,79319 & $\begin{array}{l}13,5731 \\
2.13296\end{array}$ & 0,40539 \\
\hline $\begin{array}{l}\mathrm{RS}(425 \mathrm{C}) \\
d=1\end{array}$ & $\begin{array}{c}0,22442 \\
-0.05682\end{array}$ & 0,64713 & 0,7052 & $\begin{array}{c}13,4381 \\
1.8564\end{array}$ & 0,85344 \\
\hline $\begin{array}{l}\mathrm{RS}(475 \mathrm{C}) \\
d=2\end{array}$ & $\begin{array}{c}0,27183 \\
-0.03377\end{array}$ & 0,70987 & 0,70286 & $\begin{array}{l}13,7273 \\
2.52004\end{array}$ & 0,61121 \\
\hline $\begin{array}{l}\mathrm{RS}(575 \mathrm{C}) \\
d=3\end{array}$ & $\begin{array}{c}0,12765 \\
-0.01564 \\
\end{array}$ & 0,67151 & 0,72011 & $\begin{array}{l}13,5682 \\
1.14542 \\
\end{array}$ & 0,93181 \\
\hline
\end{tabular}

In contrast with Table 1 the values in columns 5 and 6 have the nonmonotone behavior. 


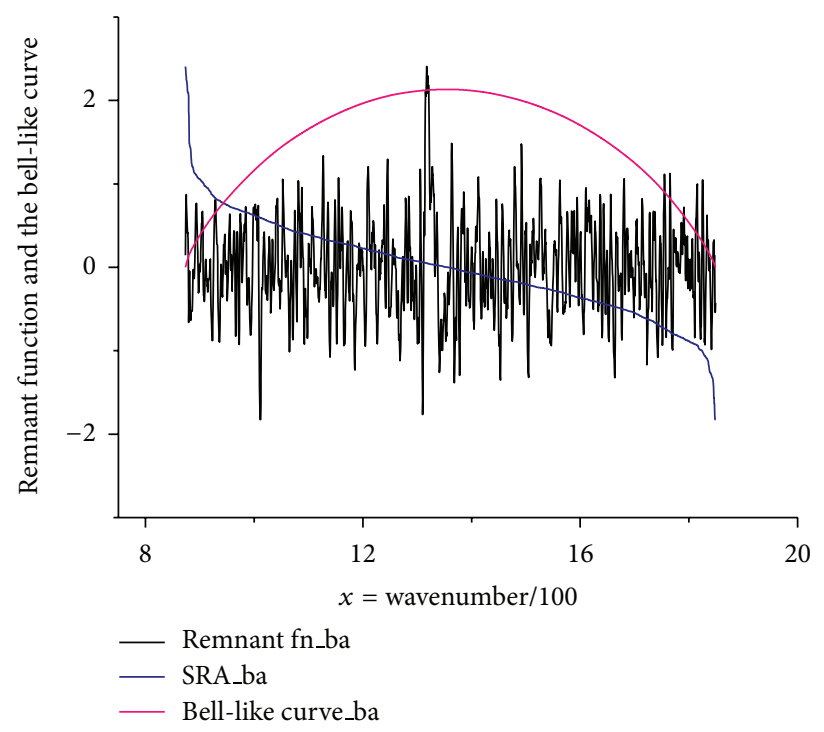

Figure 6: This plot demonstrates two steps (described in the text) that allow transforming the initial remnant function (marked by black line) for the RS $(d=0)$ to the sequence of the ranged amplitudes (SRA) and finally to the bell-like curve (marked by red solid line).

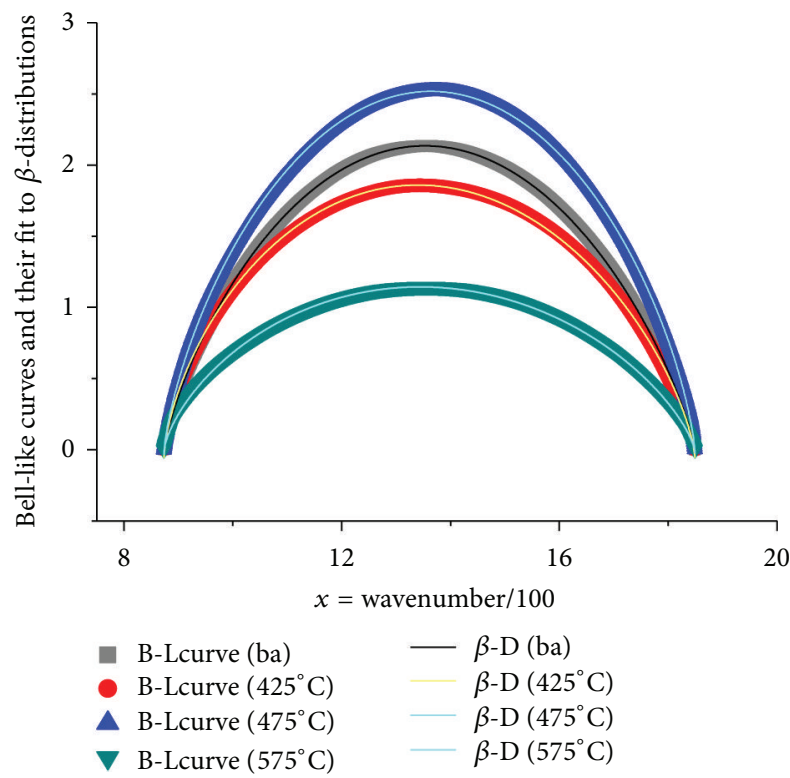

FIGURE 7: This plot shows the calculated bell-like curves and their fit to the beta-distribution function (12). The fitting parameters are collected in Table 2. One can notice that the behavior of these curves with respect to the values of the annealing temperatures is not monotone. The highest curve belongs to the annealing spectrum with $475^{\circ} \mathrm{C}$. Then the curves belonging to RS before annealing and $425^{\circ} \mathrm{C}$ have monotone behavior and the lowest curve belongs to the annealing temperature $575^{\circ} \mathrm{C}$.

noise fluctuations cannot be eliminated easily because of their quantum character. This current research (applied in the first time to nanodiamonds Raman spectra) undoubtedly merits a further research.

\section{Acknowledgments}

Raoul R. Nigmatullin and Diana Povarova express their thankfulness to the authorities of Kazan Federal University for financial support of this research. This study was funded by the Deanship of Scientific Research (DSR), King Abdulaziz University, Jeddah, Saudi Arabia, under Grant no. 903-003D1434. The authors thank the DSR for the technical and financial support.

\section{References}

[1] X. Jiang, J. Zhao, C. Zhuang, B. Wen, and X. Jiang, "Mechanical and electronic properties of ultrathin nanodiamonds under uniaxial compressions," Diamond and Related Materials, vol. 19, no. 1, pp. 21-25, 2010.

[2] M. Werner and R. Locher, "Growth and application of undoped and doped diamond films," Reports on Progress in Physics, vol. 61, no. 12, pp. 1665-1710, 1998.

[3] E. Osawa, "Recent progress and perspectives in single-digit nanodiamond," Diamond and Related Materials, vol. 16, no. 12, pp. 2018-2022, 2007.

[4] É. M. Galimov, A. M. Kudin, V. N. Skorobogatskiǐ et al., "Experimental corroboration of the synthesis of diamond in the cavitation process," Doklady Physics, vol. 49, no. 3, pp. 150-153, 2004.

[5] Y. R. Chang, H.-Y. Lee, K. Chen et al., "Mass production and dynamic imaging of fluorescent nanodiamonds," Nature Nanotechnology, vol. 3, no. 5, pp. 284-288, 2008.

[6] V. N. Mochalin and Y. Gogotsi, "Wet chemistry route to hydrophobic blue fluorescent nanodiamond," Journal of the American Chemical Society, vol. 131, no. 13, pp. 4594-4595, 2009.

[7] Y. Zhang, C. Zang, H. Ma et al., "HPHT synthesis of large single crystal diamond doped with high nitrogen concentration," Diamond and Related Materials, vol. 17, no. 2, pp. 209-211, 2008. 
[8] D. M. Gnien, "Nanocrystalline diamondfilms1," Annual Review of Materials Science, vol. 29, pp. 211-259, 1999.

[9] A. K. Dua, V. C. George, M. Friedrich, and D. R. T. Zahn, "Effect of deposition parameters on different stages of diamond deposition in HFCVD technique," Diamond and Related Materials, vol. 13, no. 1, pp. 74-84, 2004.

[10] Q. Zou, Y. G. Li, L. H. Zou, and M. Z. Wang, "Characterization of structures and surface states of the nanodiamond synthesized by detonation," Materials Characterization, vol. 60, no. 11, pp. 1257-1262, 2009.

[11] T. Jiang and K. Xu, "FTIR study of ultradispersed diamond powder synthesized by explosive detonation," Carbon, vol. 33, no. 12, pp. 1663-1671, 1995.

[12] G.-W. Yang, J.-B. Wang, and Q.-X. Liu, "Preparation of nanocrystalline diamonds using pulsed laser induced reactive quenching," Journal of Physics Condensed Matter, vol. 10, no. 35, pp. 7923-7927, 1998.

[13] J.-P. Boudou, P. A. Curmi, F. Jelezko et al., "High yield fabrication of fluorescent nanodiamonds," Nanotechnology, vol. 20, no. 23, Article ID 235602, 2009.

[14] Y. G. Gogotsi, "Structure of carbon produced by hydrothermal treatment of $\beta$-SiC powder," Journal of Materials Chemistry, vol. 6, no. 4, pp. 595-604, 1996.

[15] S. Welz, Y. Gogotsi, and M. J. McNallan, "Nucleation, growth, and graphitization of diamond nanocrystals during chlorination of carbides," Journal of Applied Physics, vol. 93, no. 7, pp. 4207-4214, 2003.

[16] T. L. Daulton, M. A. Kirk, R. S. Lewis, and L. E. Rehn, "Production of nanodiamonds by high-energy ion irradiation of graphite at room temperature," Nuclear Instruments and Methods in Physics Research B, vol. 175-177, pp. 12-20, 2001.

[17] F. Banhart and P. M. Ajayan, "Carbon onions as nanoscopic pressure cells for diamond formation," Nature, vol. 382, no. 6590, pp. 433-435, 1996.

[18] X. C. LeQuan, W. P. Kang, J. L. Davidson, M. Guo, and B. K. Choi, "Micro-Raman, SEM, XPS, and electron field emission characterizations of nitrogen-induced shallow defects on nanodiamond films fabricated with different growth parameters," Diamond and Related Materials, vol. 18, no. 2-3, pp. 191-195, 2009.

[19] A. M. Schrand, H. Huang, C. Carlson et al., "Are diamond nanoparticles cytotoxic?" Journal of Physical Chemistry B, vol. 111, no. 1, pp. 2-7, 2007.

[20] V. V. Danilenko, "On the history of the discovery of nanodiamond synthesis," Physics of the Solid State, vol. 46, no. 4, pp. 595-599, 2004.

[21] N. R. Greiner, D. S. Phillips, J. D. Johnson, and F. Volk, "Diamonds in detonation soot," Nature, vol. 333, no. 6172, pp. 440-442, 1988.

[22] M. Ozawa, M. Inaguma, M. Takahashi, F. Kataoka, A. Krüger, and E. Osawa, "Preparation and behavior of brownish, clear nanodiamond colloids," Advanced Materials, vol. 19, no. 9, pp. 1201-1206, 2007.

[23] J. R. Maze, P. L. Stanwix, J. S. Hodges et al., "Nanoscale magnetic sensing with an individual electronic spin in diamond," Nature, vol. 455 , no. 7213, pp. 644-647, 2008.

[24] A. Krueger, "Diamond nanoparticles: jewels for chemistry and physics," Advanced Materials, vol. 20, no. 12, pp. 2445-2449, 2008.

[25] W.-W. Zheng, Y.-H. Hsieh, Y.-C. Chiu, S.-J. Cai, C.-L. Cheng, and C. Chen, "Organic functionalization of ultradispersed nanodiamond: synthesis and applications," Journal of Materials Chemistry, vol. 19, no. 44, pp. 8432-8441, 2009.

[26] B. V. Spitsyn, J. L. Davidson, M. N. Gradoboev et al., "Inroad to modification of detonation nanodiamond," Diamond and Related Materials, vol. 15, no. 2-3, pp. 296-299, 2006.

[27] K. D. Behler, A. Stravato, V. Mochalin, G. Korneva, G. Yushin, and Y. Gogotsi, "Nanodiamond-polymer composite fibers and coatings," ACS Nano, vol. 3, no. 2, pp. 363-369, 2009.

[28] Q. Zhang, V. N. Mochalin, I. Neitzel et al., "Fluorescent PLLA-nanodiamond composites for bone tissue engineering," Biomaterials, vol. 32, no. 1, pp. 87-94, 2011.

[29] D. H. Wang, L.-S. Tan, H. Huang, L. Dai, and E. Osawa, "Insitu nanocomposite synthesis: arylcarbonylation and grafting of primary diamond nanoparticles with a poly(ether-ketone) in polyphosphoric acid," Macromolecules, vol. 42, no. 1, pp. 114124, 2009.

[30] J. Cheng, J. He, C. Li, and Y. Yang, "Facile approach to functionalize nanodiamond particles with V-shaped polymer brushes," Chemistry of Materials, vol. 20, no. 13, pp. 4224-4230, 2008.

[31] V. N. Mochalin, I. Neitzel, B. J. M. Etzold, A. Peterson, G. Palmese, and Y. Gogotsi, "Covalent incorporation of aminated nanodiamond into an epoxy polymer network," ACS Nano, vol. 5, no. 9, pp. 7494-7502, 2011.

[32] R. A. Shimkunas, E. Robinson, R. Lam et al., "Nanodiamondinsulin complexes as $\mathrm{pH}$-dependent protein delivery vehicles," Biomaterials, vol. 30, no. 29, pp. 5720-5728, 2009.

[33] K. V. Purtov, A. I. Petunin, A. E. Burov, A. P. Puzyr, and V. S. Bondar, "Nanodiamonds as carriers for address delivery of biologically active substances," Nanoscale Research Letters, vol. 5, no. 3, pp. 631-636, 2010.

[34] A. Alhaddad, M.-P. Adam, J. Botsoa et al., "Nanodiamond as a vector for siRNA delivery to Ewing sarcoma cells," Small, vol. 7, no. 21, pp. 3087-3095, 2011.

[35] A. Schrand, J. Johnson, L. Dai et al., "Cytotoxicity and genotoxicity of carbon nanomaterials," in Safety of Nanoparticles, T. J. Webster, Ed., pp. 159-187, Springer, New York, NY, USA, 2009.

[36] A. M. Schrand, S. A. C. Hens, and O. A. Shenderova, "Nanodiamond particles: properties and perspectives for bioapplications," Critical Reviews in Solid State and Materials Sciences, vol. 34, no. 1-2, pp. 18-74, 2009.

[37] C.-C. Fu, H.-Y. Lee, K. Chen et al., "Characterization and application of single fluorescent nanodiamonds as cellular biomarkers," Proceedings of the National Academy of Sciences of the United States of America, vol. 104, no. 3, pp. 727-732, 2007.

[38] S.-J. Yu, M.-W. Kang, H.-C. Chang, K.-M. Chen, and Y.-C. Yu, "Bright fluorescent nanodiamonds: no photobleaching and low cytotoxicity," Journal of the American Chemical Society, vol. 127, no. 50, pp. 17604-17605, 2005.

[39] L.-C. L. Huang and H.-C. Chang, "Adsorption and immobilization of cytochrome c on nanodiamonds," Langmuir, vol. 20, no. 14, pp. 5879-5884, 2004.

[40] X. Kong, L. C. L. Huang, S.-C. V. Liau, C.-C. Han, and H.-C. Chang, "Polylysine-coated diamond nanocrystals for MALDITOF mass analysis of DNA oligonucleotides," Analytical Chemistry, vol. 77, no. 13, pp. 4273-4277, 2005.

[41] J. R. Mansfield, K. W. Gossage, C. C. Hoyt, and R. M. Levenson, "Autofluorescence removal, multiplexing, and automated analysis methods for in-vivo fluorescence imaging," Journal of Biomedical Optics, vol. 10, no. 4, Article ID 041207, 2005. 
[42] Z. Y. Lien, T. C. Hsu, K. K. Liu, W. S. Liao, K. C. Hwang, and J. I. Chao, "Cancer cell labeling and tracking using fluorescent and magnetic nanodiamond," Biomaterials, vol. 33, no. 26, pp. 6172-6185, 2012.

[43] S. Michaelson and A. Hoffman, "Hydrogen bonding, content and thermal stability in nano-diamond films," Diamond and Related Materials, vol. 15, no. 4-8, pp. 486-497, 2006.

[44] Y. Tzeng and Y. K. Liu, "Diamond CVD by microwave plasmas in argon-diluted methane without or with $2 \%$ hydrogen additive," Diamond and Related Materials, vol. 14, no. 3-7, pp. 261265, 2005.

[45] C.-M. Băleanu, R. R. Nigmatullin, S. S. Cetin et al., "New method and treatment technique applied to interband transition in $\mathrm{GaAs}_{1-x} \mathrm{P}_{x}$ ternary alloys," Central European Journal of Physics, vol. 9, no. 3, pp. 729-739, 2011.

[46] M. L. Ciurea, S. Lazanu, I. Stavarache et al., "Stress-induced traps in multilayered structures," Journal of Applied Physics, vol. 109, no. 1, Article ID 013717, 2011.

[47] R. R. Nigmatullin, C. Ionescu, and D. Baleanu, "NIMRAD: novel technique for respiratory data treatment," Signal, Image and Video Processing, pp. 1-16, 2012.

[48] R. R. Nigmatullin, C.-M. Ionescu, S. Osokin et al., "Noninvasive methods applied for complex signals," Romanian Reports in Physics, vol. 64, no. 4, pp. 1032-1045, 2012.

[49] R. R. Nigmatullin, "The statistics of the fractional moments: is there any chance to "read quantitatively" any randomness?" Signal Processing, vol. 86, no. 10, pp. 2529-2547, 2006.

[50] R. R. Nigmatullin, A. A. Arbuzov, S. O. Nelson, and S. Trabelsi, "Dielectric relaxation in complex systems: quality sensing and dielectric properties of honeydew melons from $10 \mathrm{MHz}$ to 1.8 GHz," Journal of Instrumentation, vol. 1, no. 1, pp. 1-20, 2006.

[51] R. R. Nigmatullin, "Universal distribution function for the strongly-correlated fluctuations: general way for description of different random sequences," Communications in Nonlinear Science and Numerical Simulation, vol. 15, no. 3, pp. 637-647, 2010.

[52] R. R. Nigmatullin, "Recognition of nonextensive statistical distributions by the eigencoordinates method," Physica A, vol. 285, no. 3, pp. 547-565, 2000. 


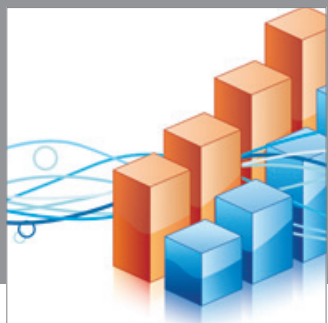

Advances in

Operations Research

mansans

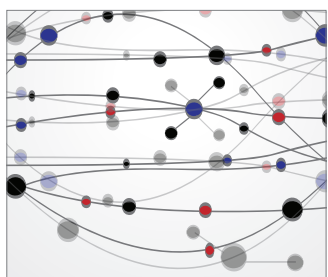

The Scientific World Journal
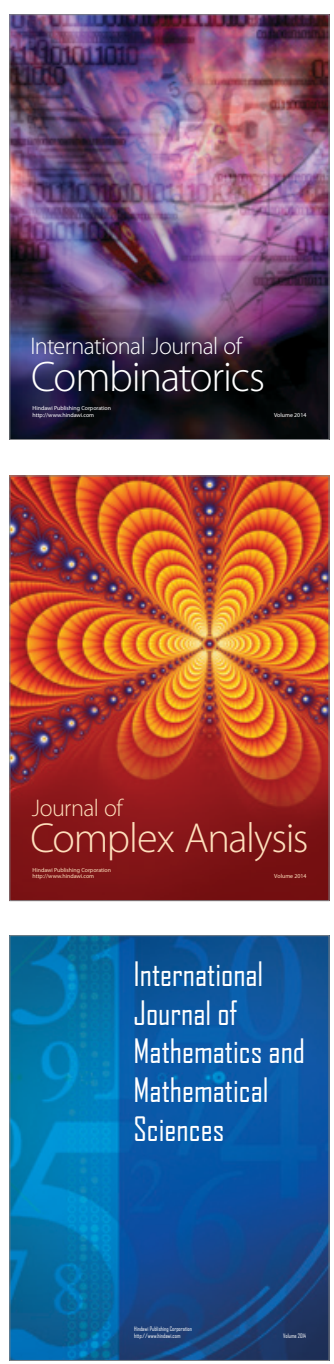
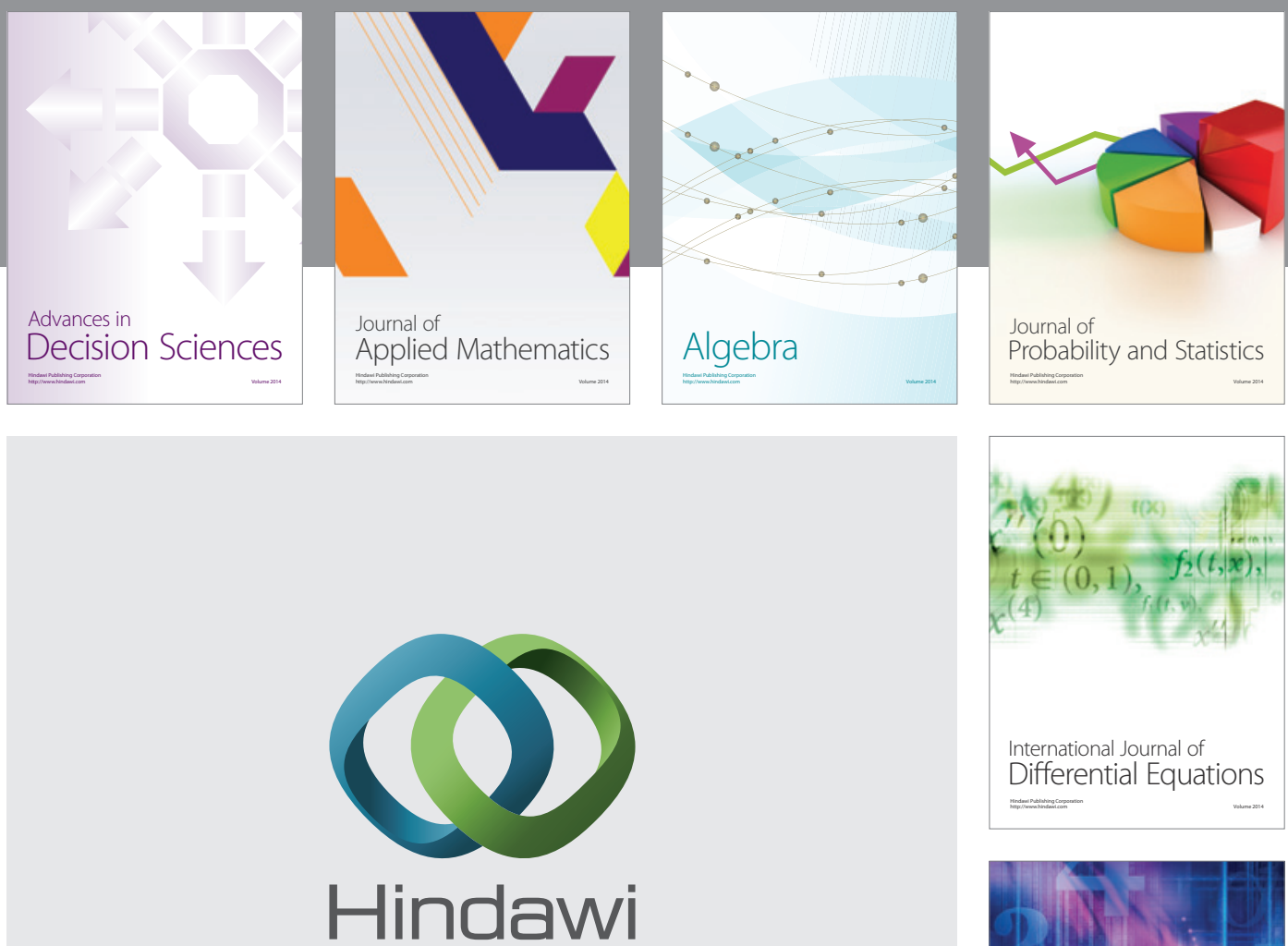

Submit your manuscripts at http://www.hindawi.com
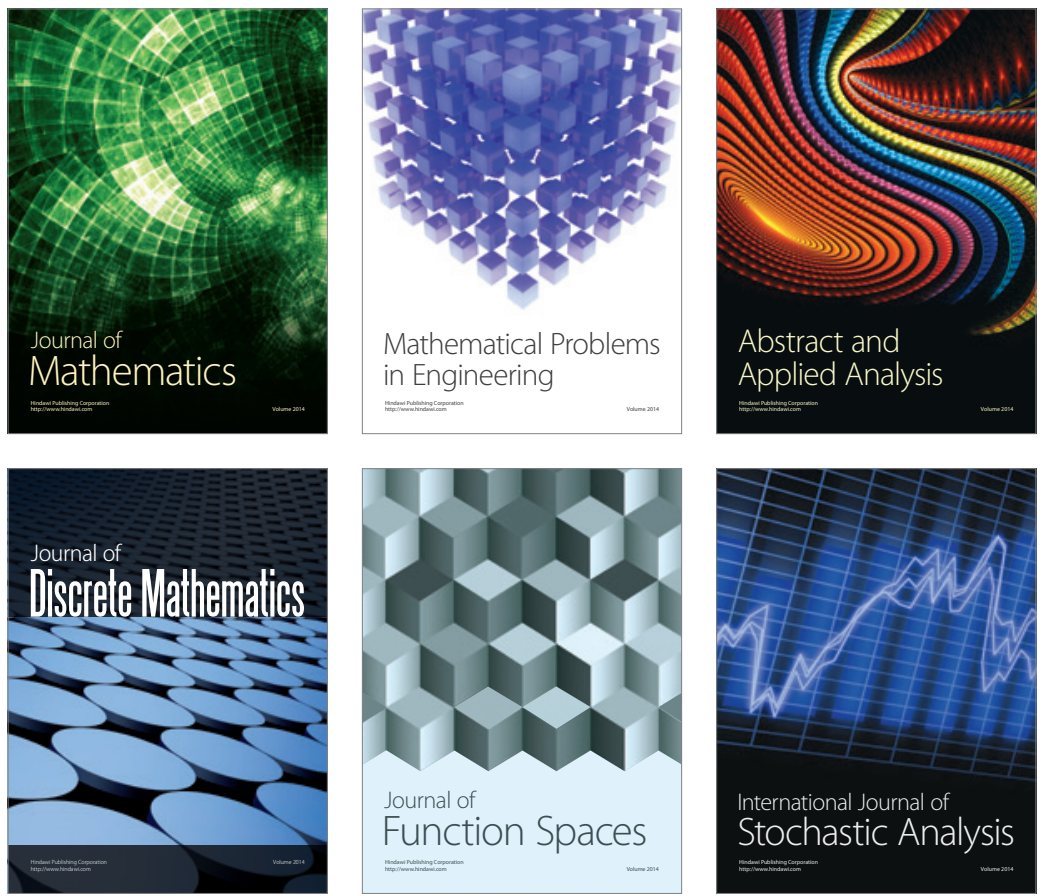

Journal of

Function Spaces

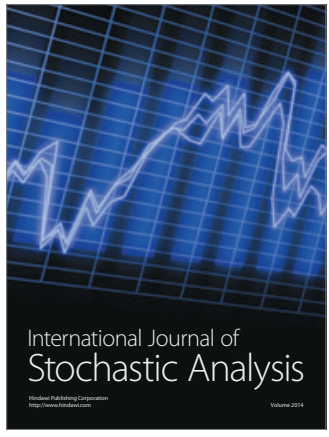

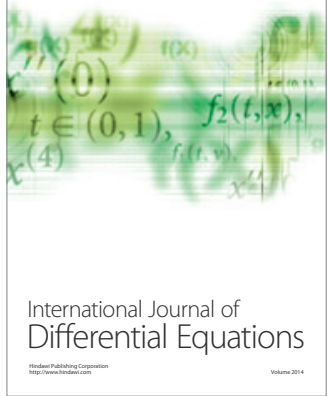
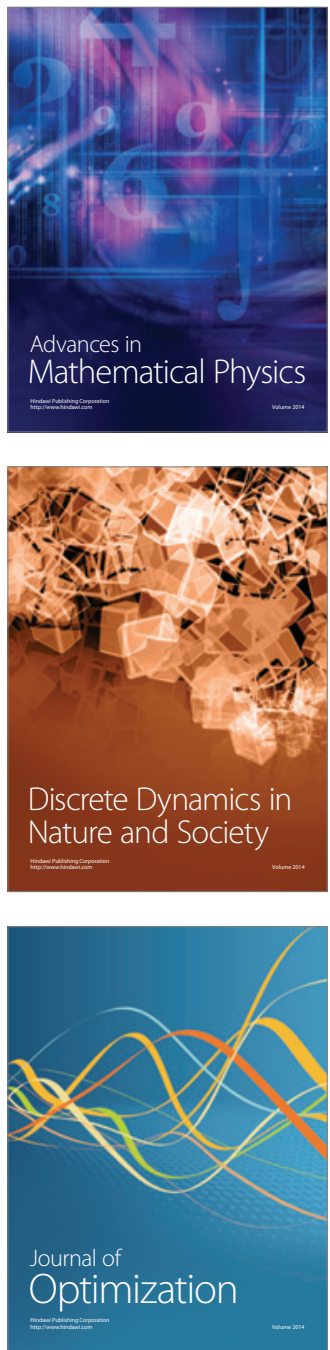